\title{
Inflammatory response following neutrophil recovery postchemotherapy in acute myeloid leukemia cases without evidence of infection: role of homing of neutrophils
}

\author{
This article was published in the following Dove Press journal: \\ Journal of Blood Medicine \\ 10 March 2014 \\ Number of times this article has been viewed
}

\author{
Rahul D Pawar' \\ Travis Williams ${ }^{2}$ \\ Rahul Khera ${ }^{2}$ \\ Albert Eid ${ }^{3}$ \\ Omar S Aljitawi ${ }^{1,4}$ \\ Reginald W Dusing 5 \\ 'Internal Medicine, ${ }^{2}$ Hematology/ \\ Oncology, ${ }^{3}$ nfectious Diseases, \\ ${ }^{4}$ Pathology and Laboratory Medicine, \\ ${ }^{5}$ Radiology, University of Kansas \\ Medical Center, Kansas City, KS, USA
}

Correspondence: Rahul D Pawar Internal Medicine, University of Kansas Medical Center, 390I Rainbow Boulevard, Kansas City, KS 66160, USA Email rpawar@kumc.edu

\begin{abstract}
Neutropenic sepsis is a common clinical entity occurring in postchemotherapy patients. Infection may not be the cause of fever in such patients after neutrophil-count recovery. Herein, we present two patients who developed fever during the neutropenic phase of induction chemotherapy and were treated with broad-spectrum antibiotics until they were no longer febrile and had recovered their neutrophil count. Being off antibiotics, they redeveloped fever within 48-72 hours. These fevers seemed to be secondary to postinfectious inflammatory response and not infection, supported by the fact that adequate antibiotic treatment was given and the collected fluid contained neutrophils but the cultures were negative. We hypothesize an explanation for this phenomenon based on the "homing of neutrophils" to bone marrow, which involves chemoattraction of CXC chemokine receptor (CXCR)-4 expressed on neutrophils towards the chemokine stromal cell-derived factor (SDF)-1 (CXCL12) expressed constitutively by bone marrow. Literature has shown that elevation of SDF-1 levels at injured/inflamed sites might create a similar gradient. This gradient results in the migration of neutrophils to the sites of previous injury/inflammation, leading to the formation of sterile abscesses. Based on our cases, we also conclude that antibiotics do not prevent the formation or treat such sterile "abscesses"; however, the drainage of these "abscesses" and treatment with anti-inflammatory agents are useful in such cases.
\end{abstract}

Keywords: SDF-1, CXCR4, postinfectious inflammatory response, homing of neutrophils

\section{Introduction}

Neutrophils are one of the first cells arriving at the site of infection, and play an important role in combating an invading pathogen. ${ }^{1}$ Neutrophils express $\mathrm{CXC}$ chemokine receptor (CXCR)-4 on their cell surface, ${ }^{2,3}$ whose expression is upregulated during maturation. ${ }^{3,4}$ As they mature, the upregulation of CXCR4 receptors enables them to migrate down the concentration gradient of stromal cell-derived factor (SDF)-1. This chemoattraction of CXCR4 receptors to SDF-1 forms a CXCR4/CXCL12 axis., Bone marrow, for example, expresses SDF-1 constitutively. ${ }^{2,3}$ Thus, during homing of neutrophils to bone marrow, they migrate towards SDF-1 expressed by bone marrow, forming an important mechanism for neutrophil homing to bone marrow. ${ }^{2,3}$ The literature documents elevated SDF-1 levels at the sites of injury/inflammation in lungs, 5,6 intestine, ${ }^{7,8}$ synovial fluid, ${ }^{7,9}$ brain, ${ }^{10-12}$ kidney, ${ }^{13}$ heart,,${ }^{14}$ and liver. ${ }^{15}$ This increase of SDF-1 in such sites leads to chemotaxis of neutrophils to sites of injury/inflammation, sometimes leading to unwanted sequelae. Herein, we present two cases with 
adequately treated localized infectious foci while neutropenic secondary to induction chemotherapy, who 48-72 hours after neutrophil recovery developed systemic inflammatory response syndrome (SIRS) with aseptic inflammatory collections in the same region of previous inflammatory foci. We also provide an explanation for this phenomenon based on neutrophil homing to areas of prior inflammation possibly driven by CXCR4 and SDF-1 interaction, and use this knowledge in proposing treatment recommendations for such inflammatory responses.

\section{Case presentation}

A 32-year-old woman with remote history of pilonidal abscess presented with easy bruising, fatigue, generalized bony pain, and heavy menses. She was found to have acute myeloid leukemia (AML) with 34\% bone marrow blasts and $\mathrm{t}(8 ; 21)$ (q22;q22). She was placed on levaquin, acyclovir, and posaconazole for prophylaxis, and induction chemotherapy with standard daunorubicin and cytarabine was initiated for a total of 7 days. While neutropenic, she developed an area of tenderness and erythema around her sacrum in the previous site of a pilonidal cyst and then became febrile. Cefepime was initiated, and was later changed to vancomycin and piperacillin/ tazobactam because of suspected rash from cefepime. She remained febrile for 3 days after initiating antibiotic therapy. Blood cultures were drawn and remained negative. Computed tomography (CT) of the pelvis revealed ill-defined soft-tissue thickening in the mid-lower back from the lumbar spine to the sacrum within the deep subcutaneous fat to the gluteal crease without any evidence of muscular involvement. There was also a well-defined fluid collection measuring approximately $2.9 \times 0.8 \mathrm{~cm}$ within the subcutaneous tissues, superficial to the lower sacrum and to the right of the midline (Figure 1A). Erythema and fever resolved, and tenderness
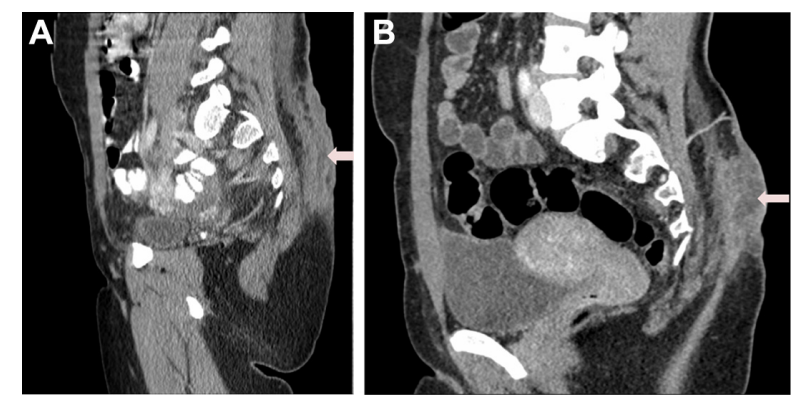

Figure I Pilonidal abscess, sagittal computed tomography (CT) images on admission (A) and time of neutrophil recovery (B). (A) CT scan showing soft-tissue ill-defined thickening from the lumbar spine to the sacrum, with a focal well-defined fluid collection measuring approximately $2.9 \times 0.8 \mathrm{~cm}$ (arrow). (B) Three weeks postinduction CT sagittal images showing increase in well-defined fluid collection, now measuring $1.3 \times 2.2 \times 6.4 \mathrm{~cm}$ (marked with arrow). improved with antibiotics. Vancomycin was discontinued after 48 hours, but piperacillin/tazobactam was continued until her neutrophil count became $>500$ neutrophils $/ \mu \mathrm{L}$. The total duration of antibiotic therapy was 11 days. A postinduction bone marrow evaluation revealed no residual leukemia.

After 48 hours off antibiotics, the presacral tenderness and redness returned. Examination revealed an area of fluctuance, erythema, and tenderness in the same location of her previous pilonidal cystectomy. At this time, her absolute neutrophil count (ANC) was $2,500 / \mu \mathrm{L}$. CT of the pelvis showed an increase in size of well-defined fluid collection, measuring $1.3 \times 2.2 \times 6.4 \mathrm{~cm}$ (Figure $1 \mathrm{~B}$ ). She was off antibiotics for 48-72 hours before aspiration, and drainage was performed. Foul, cloudy, purulent fluid was aspirated and sent for analysis. This showed many neutrophils with no organisms, and cultures were negative. The patient was placed on ertapenem, and her symptoms improved primarily after initial drainage. Follow-up sonogram, done after 3 days, showed a mild complex fluid collection within the subcutaneous tissues. She underwent incision and drainage of the area of greatest redness. Retrieved tissue and fluid were sent for cultures and remained negative again. Gram stain of the swabbed fluid showed rare neutrophils, and no organisms were seen. The area healed well, and after 12 days she proceeded with treatment for her AML with a matched-sibling donor stem cell transplant without complications.

The second case was a 52-year-old male patient who presented with pancytopenia, $35 \%$ myeloid blasts in the bone marrow, fever, chills, and tachycardia, with redness, swelling, and pain involving the medial posterior left thigh, and mild redness and swelling but no pain of the lateral aspect of the right thigh. Magnetic resonance imaging (MRI) of the left thigh showed pyomyositis with extensive edema throughout quadriceps musculature, with focal areas of myonecrosis and intercommunicating pockets of fluid. Creatine phosphokinase was $905 \mathrm{U} / \mathrm{L}$. There was also subcutaneous edema suggestive of cellulitis. He was diagnosed with AML and sepsis. Peripheral blood, bone marrow, and urine cultures obtained initially showed no bacterial or fungal infection. He was started on vancomycin, cefepime, and clindamycin, and then changed to vancomycin and piperacillin/tazobactam after 8 days. AML induction therapy with daunorubicin and cytarabine was initiated for a total of 7 days. Surgical intervention was considered but not done, due to clinical improvement while on antibiotics.

With antibiotic therapy, the patient became afebrile with decreased pain, swelling, and redness of the left upper thigh and stabilization of the right thigh. On postinduction day 21, 
the ANC was $80 / \mu \mathrm{L}$, and while on piperacillin/tazobactam, the patient developed fever. A repeat MRI showed progressively evolving areas of myonecrosis/abscess in the left thigh, predominately in the abductor brevis and magnus, and unchanged to slightly decreased area of myonecrosis/ abscess in the proximal right thigh, with slightly increased enhancement and edema in the adductor magnus (Figure 2A). Orthopedic surgery was reconsidered, and it was thought that overall the abscesses were unchanged. Blood cultures were drawn and were negative. Slowly, his symptoms improved with antibiotics, and he was discharged off antibiotics after he became afebrile and had recovered his neutrophil count (ANC was 666/ $\mathrm{L} \mathrm{L}$ upon discontinuation of antibiotics).

Three days later, he presented with severe pain and increased swelling in his left thigh. His white blood cell count was $8,800 / \mu \mathrm{L}$ at that point. On examination, his left thigh was swollen and painful to the touch without any fluctuation. A repeat MRI showed marked progression and enlargement of multiple fluid collections in the left thigh consistent with worsening areas of myonecrosis/abscess (Figure 2B). Routine blood cultures and one fungal blood culture were obtained, and showed no growth. Prior to reinitiation of antibiotic therapy, a CT-guided aspiration was done and a drain placed. The recovered fluid showed no bacteria, fungus, or mycobacteria. Gram stain, however, showed many neutrophils and red blood cells, but no organisms. Following the procedure, his left thigh pain improved; however, his pain dramatically improved with anti-inflammatory agents. Vancomycin and piperacillin/tazobactam were started, and later changed to ertapenem. The drain output slowed down and became insignificant 15 days post-drain placement. Ertapenem was stopped 20 days after drain placement. The patient remained afebrile and proceeded with subsequent chemotherapy.

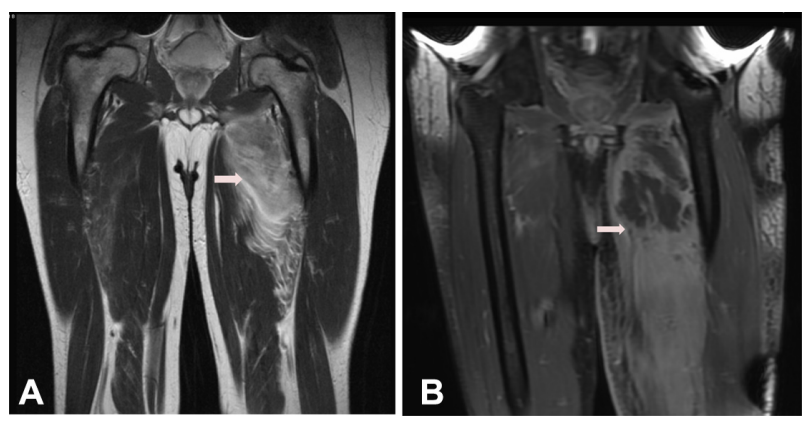

Figure 2 (A) Magnetic resonance imaging (MRI) T2 coronal images with marked edema and enhancement of the left adductor magnus and brevis muscles, with focal areas of nonenhancement consistent with myonecrosis (arrow). (B) MRI TI fast-spin coronal images with progression of the edema and development of a fluid collection in the proximal medial thigh measuring $3.9 \times 10.3 \times 8.5 \mathrm{~cm}$ (marked with arrow).

\section{Discussion}

Migration of cells through the blood to the bone marrow niches requires active navigation, a process termed "homing." Homing is thought to be a coordinated, multistep process that involves signaling bySDF-1 and its chemokine receptor CXCR4, as well as other chemokines. ${ }^{2-4}$ Homing is essential for bone marrow stem cell transplantation to be effective, but is also essential for successful host eradication of bacterial pathogens and survival in polymicrobial sepsis. ${ }^{16}$ The bone marrow acts as a reservoir for neutrophils, whose release and egress is tightly regulated. ${ }^{2,3}$ This interaction is very crucial during bacterial infection and sepsis, but is also very important in sterile inflammatory processes.

A sterile inflammatory process happens in times of vascular disruption, not from infection but from trauma, hypotension, myocardial infarction, cerebral vascular events, or arterial thrombus. With aggressive treatment measures, such as volume replacement, revascularization, or antithrombotic therapy, the tissue that was ischemic becomes perfused again. Reperfusion injury has been studied for more than 30 years, specifically in cerebral ischemia, ${ }^{10,17}$ druginduced hepatotoxicity, ${ }^{18}$ and ischemic cardiac disease. ${ }^{14}$ Several studies have looked at the mechanism of neutrophil depletion $^{16,18}$ to prevent reperfusion injury, while others have looked at ways to alter the signaling of the SDF-1/CXCR4 axis. ${ }^{11,14,19}$

This has a direct correlation with the two cases presented, in which induction chemotherapy was given to two patients with localized inflammation. Postinduction, they developed fevers and localized infectious foci, which were appropriately treated with antibiotics. The bone marrow reserves of neutrophils, progenitor stem cells, and myeloid blasts were dramatically reduced, because of the cytotoxic doses of chemotherapy. During this phase, these two patients had resolution of their symptoms because of appropriate use of antibiotics. Standard treatment is to discontinue all antibiotics after ANC is greater than $500 / \mu \mathrm{L}$, provided there is resolution of fever and signs and symptoms of infection. In both instances, these two patients returned within 48-72 hours with $\mathrm{ANC}>2,500 / \mu \mathrm{L}$, with worsened pain and an increased fluid collection. In both cases, no organism was found, but only abundant neutrophils were seen on Gram stain. Also, in the second case, the patient's symptoms did not improve until anti-inflammatory agents were given.

One of the proposed hypotheses could be that these two patients had an infected abscess or leukemic infiltration within previous inflammatory sites when they developed fevers. This could have led to tissue ischemia or could have 
elicited inflammatory response leading to tissue injury. With this ischemia ${ }^{10-13}$ or inflammation, ${ }^{5-8}$ we hypothesize that there might have been increased levels of chemokines, specifically SDF-1, at these inflammatory sites. Antibiotics were given, and these areas were sterilized. As the bone marrow recovered, the young immature neutrophils, which are low in CXCR4 expression, started expressing CXCR4 as they matured. ${ }^{3,4}$ As they expressed CXCR4 on their surface, they acquired the ability to migrate towards elevated SDF-1 levels at previous inflammatory sites. ${ }^{3,4}$ This insurgence of neutrophils into these areas could explain in our situation the inflammatory response with increased fluctuation and return of symptoms.

It is difficult to differentiate SIRS without infection from sepsis clinically. ${ }^{20,21}$ SIRS criteria are moderately associated with infection, ${ }^{22}$ and as mortality increases with delay in administration of antibiotics, ${ }^{23}$ most cases with SIRS end up being on antibiotics. In our case as well, antibiotics were initiated but later discontinued when cultures were negative. Though some studies suggest anti-inflammatory properties of some antibiotics like macrolides and newer-generation quinolones, ${ }^{24}$ there are no definitive data to show a rationale for using antibiotics in SIRS without infection. Also, in our second case, symptoms improved only after administration of nonsteroidal anti-inflammatory drugs (NSAIDs). Though some studies have shown efficacy of NSAIDs in SIRS without infection, ${ }^{25}$ use of NSAIDs in postinfectious inflammatory response has not been studied.

In conclusion, we present two cases of a sterile inflammatory collection developing at the time of neutrophil recovery from neutropenia at the site of adequately treated infection. We speculate that these sterile inflammatory collections were related to neutrophil recovery and homing to areas of prior injury/inflammation and not in response to an active infection.

\section{Disclosure}

The authors report no conflicts of interest in this work.

\section{References}

1. Bouma G, Ancliff PJ, Thrasher AJ, Burns SO. Recent advances in the understanding of genetic defects of neutrophil number and function. Br J Haematol. 2010;151:312-326.

2. Suratt BT, Petty JM, Young SK, et al. Role of the CXCR4/SDF-1 chemokine axis in circulating neutrophil homeostasis. Blood. 2004;104:565-571.

3. Martin C, Burdon PC, Bridger G, Gutierrez-Ramos JC, Williams TJ, Rankin SM. Chemokines acting via CXCR2 and CXCR4 control the release of neutrophils from the bone marrow and their return following senescence. Immunity. 2003;19:583-593.
4. Nagase H, Miyamasu M, Yamaguchi M, et al. Cytokine-mediated regulation of CXCR4 expression in human neutrophils. J Leukoc Biol. 2002;71:711-717.

5. Petty JM, Sueblinvong V, Lenox CC, et al. Pulmonary stromal-derived factor-1 expression and effect on neutrophil recruitment during acute lung injury. J Immunol. 2007;178:8148-8157.

6. Yamada M, Kubo H, Kobayashi S, et al. The increase in surface CXCR4 expression on lung extravascular neutrophils and its effects on neutrophils during endotoxin-induced lung injury. Cell Mol Immunol. 2011;8:305-314.

7. Werner L, Guzner-Gur H, Dotan I. Involvement of CXCR4/CXCR7/ CXCL12 interactions in inflammatory bowel disease. Theranostics. 2013;3:40-46.

8. Mikami S, Nakase H, Yamamoto S, et al. Blockade of CXCL12/CXCR4 axis ameliorates murine experimental colitis. $J$ Pharmacol Exp Ther. 2008;327:383-392.

9. Xu Q, Sun XC, Shang XP, Jiang HS. Association of CXCL12 levels in synovial fluid with the radiographic severity of knee osteoarthritis. J Investig Med. 2012;60:898-901.

10. Hill WD, Hess DC, Martin-Studdard A, et al. SDF-1 (CXCL12) is upregulated in the ischemic penumbra following stroke: association with bone marrow cell homing to injury. J Neuropathol Exp Neurol. 2004;63:84-96.

11. Huang J, Li Y, Tang Y, Tang G, Yang GY, Wang Y. CXCR4 antagonist AMD3100 protects blood-brain barrier integrity and reduces inflammatory response after focal ischemia in mice. Stroke. 2013;44: 190-197.

12. Miller JT, Bartley JH, Wimborne HJ, et al. The neuroblast and angioblast chemotaxic factor SDF-1 (CXCL12) expression is briefly up regulated by reactive astrocytes in brain following neonatal hypoxic-ischemic injury. BMC Neurosci. 2005;6:63.

13. Togel F, Isaac J, Hu Z, Weiss K, Westenfelder C. Renal SDF-1 signals mobilization and homing of CXCR4-positive cells to the kidney after ischemic injury. Kidney Int. 2005;67:1772-1784.

14. Ghadge SK, Muhlstedt S, Ozcelik C, Bader M. SDF- $1 \alpha$ as a therapeutic stem cell homing factor in myocardial infarction. Pharmacol Ther. 2011;129:97-108.

15. Hassanshahi G, Jafarzadeh A, James Dickson A. Expression of stromal derived factor alpha (SDF-1 alpha) by primary hepatocytes following isolation and heat shock stimulation. Iran J Allergy Asthma Immunol. 2008;7:61-68.

16. Delano MJ, Kelly-Scumpia KM, Thayer TC, et al. Neutrophil mobilization from the bone marrow during polymicrobial sepsis is dependent on CXCL12 signaling. J Immunol. 2011;187: 911-918.

17. Kochanek PM, Hallenbeck JM. Polymorphonuclear leukocytes and monocytes/macrophages in the pathogenesis of cerebral ischemia and stroke. Stroke. 1992;23:1367-1379.

18. Liu ZX, Han D, Gunawan B, Kaplowitz N. Neutrophil depletion protects against murine acetaminophen hepatotoxicity. Hepatology. 2006;43:1220-1230.

19. Li Y, Qu J, Shelat H, Gao S, Wassler M, Geng YJ. Clusterin induces CXCR4 expression and migration of cardiac progenitor cells. Exp Cell Res. 2010;316:3435-3442.

20. Mitaka C. Clinical laboratory differentiation of infectious versus noninfectious systemic inflammatory response syndrome. Clin Chim Acta. 2005;351:17-29.

21. Nierhaus A, Linssen J, Wichmann D, Braune S, Kluge S. Use of a weighted, automated analysis of the differential blood count to differentiate sepsis from non-infectious systemic inflammation: the intensive care infection score (ICIS). Inflamm Allergy Drug Targets. 2012;11: 109-115.

22. Comstedt P, Storgaard M, Lassen AT. The systemic inflammatory response syndrome (SIRS) in acutely hospitalised medical patients: a cohort study. Scand J Trauma Resusc Emerg Med. 2009; $17: 67$. 
23. Siddiqui S, Salahuddin N, Raza A, Razzak J. How early do antibiotics have to be to impact mortality in severe sepsis? A prospective, observational study from an emergency department. J Ayub Med Coll Abbottabad. 2009;21:106-110.

24. Kawai S. [Modulation of SIRS]. Rinsho Byori. 2000;48:516-520. Japanese.
25. Havlícek K, Motycka V, Siller J, Cervinka V. Systemic inflammatory response syndrome (SIRS) in serious chest injuries: is a pharmacological blockade effective? Ann Thorac Cardiovasc Surg. 2005;11: 232-237.

\section{Publish your work in this journal}

The Journal of Blood Medicine is an international, peer-reviewed, open access, online journal publishing laboratory, experimental and clinical aspects of all topics pertaining to blood based medicine including but not limited to: Transfusion Medicine; Blood collection, Donor issues, Transmittable diseases, and Blood banking logistics; Immunohematology; Artificial and alternative

\section{Dovepress}

blood based therapeutics; Hematology; Biotechnology/nanotechnology of blood related medicine; Legal aspects of blood medicine; Historical perspectives. The manuscript management system is completely online and includes a very quick and fair peer-review system. Visit http://www.dovepress.com/ testimonials.php to read real quotes from published authors.

Submit your manuscript here: http://www.dovepress.com/Journal-of-blood-medicine-journal 